\title{
Tryptophan depletion disinhibits punishment but not reward prediction: implications for resilience
}

\author{
Oliver J. Robinson • Roshan Cools • \\ Barbara J. Sahakian
}

Received: 11 May 2011 / Accepted: 17 June 2011 / Published online: 19 July 2011

(C) The Author(s) 2011. This article is published with open access at Springerlink.com

\begin{abstract}
Rationale We have previously shown that tryptophan depletion enhances punishment but not reward prediction (Cools et al. in Neuropsychopharmacology 33:2291-2299, 2008b). This provided evidence for a valence-specific role of serotonin (which declines under depleted tryptophan) in aversive processing. Recent theoretical (Dayan and Huys in PLoS Comput Biol 4:e4, 2008) and experimental (Crockett et al. in J Neurosci 29:11993-11999, 2009) approaches have, however, further specified this role by showing that serotonin is critical for punishment-induced inhibition.

Objectives We sought to examine the role of serotonin in punishment-induced inhibition. We also examined the impact of induced mood on this effect to assess whether effects of tryptophan depletion on affective inhibition are moderated by mood.

Methods Healthy females consumed a balanced amino acid mixture with $(N=20)$ or without $(N=21)$ the serotonin precursor tryptophan. Each subject completed either negative
\end{abstract}

O. J. Robinson · B. J. Sahakian

Department of Psychiatry and MRC/Wellcome Trust Behavioural and Clinical Neuroscience Institute, University of Cambridge,

Cambridge, Addenbrooke's Hospital,

P.O. Box 189, Level E4, Hills Road,

Cambridge CB2 2QQ, UK

\section{O. J. Robinson ( $\varangle)$}

National Institute of Mental Health, National Institutes of Health, Bethesda, MD, USA

e-mail: oliver.j.robinson@gmail.com

R. Cools

Donders Institute for Brain, Cognition and Behaviour,

Centre for Cognitive Neuroimaging, Department of Psychiatry,

Radboud University Nijmegen Medical Centre,

Nijmegen, The Netherlands or neutral mood induction. All subjects completed the reward and punishment reversal learning task adopted in the previous study.

Results We demonstrate a punishment prediction impairment in individuals who consumed tryptophan which was absent in individuals who were depleted of tryptophan. This effect was impervious to mood state.

Conclusions Our results suggest that serotonin promotes the inhibition of responses to punishing outcomes. This may lead to reduced punishment prediction accuracy in the presence of tryptophan and may contribute to resilience to affective disorders. Reduction of serotonin via tryptophan depletion then removes this inhibition. As such, we highlight a mechanism by which reduced serotonin can contribute to disorders of impulsivity and compulsivity as well as disorders of emotion.

Keywords Serotonin $\cdot$ Inhibition $\cdot$ Reward $\cdot$ Punishment . Resilience

\section{Introduction}

Serotonin has long been implicated in aversive processing. Individuals with enhanced aversive processing, such as those with depression or anxiety, exhibit altered serotonin function (Deakin and Graeff 1991), and serotonin has been argued to provide an aversive opponent to dopamine-related appetitive responses (Boureau and Dayan 2011; Cools et al. 2011; Daw et al. 2002). In 2008, we published a paper (Cools et al. 2008b) demonstrating that acute tryptophan depletion (ATD), associated with global reduction in central serotonin levels (Crockett et al. in submission; Gessa et al. 1974; Young and Gauthier 1981), enhanced punishment, but not reward prediction in healthy individuals. These 
findings provided clear experimental evidence for a valence-specific role of serotonin in aversive processing.

However, serotonin also plays a key role in reducing motor output and behavioural inhibition (Soubrié 1986), and it has been argued that aversive processing and inhibition cannot easily be separated (Cools et al. 2011; Dayan and Huys 2008). The prepotent response to aversive stimuli across the animal kingdom, for instance is behavioural inhibition and withdrawal (Dayan and Huys 2009). A recent computational model therefore sought to formally define this relationship (Dayan and Huys 2008). In this model, serotonin acts to inhibit punishment-associated internally directed thoughts, thus pruning away aversive branches of a Markov decision tree. Recent experimental findings have provided support for this model (Crockett et al. 2009). Using a novel task designed to disentangle inhibition and aversive responses, Crockett et al. showed that tryptophan depletion had no effect on (1) general motor inhibition or (2) sensitivity to aversive outcomes, but did (3) selectively reduce punishment-induced inhibition. As such, serotonin was critical for neither punishment processing, nor behavioural inhibition alone, but was specifically critical for punishment-induced inhibition.

In light of these recent studies, we sought to extend our previous findings by increasing the sample size and by examining the impact of mood state on the role of serotonin in punishment-induced inhibition. Specifically, recent reviews have sought to cast doubt over the effects of ATD on central serotonin transmission (Feenstra and van der Plasse 2010; van Donkelaar et al. 2011) (but see also (Crockett et al. in submission; Gessa et al. 1974; Williams et al. 1999)). Replicating our previous valence-specific effect, which mimics the effects of other more direct serotonin manipulations (Cools et al. 2008a; Crockett et al. in submission), in a separate and larger sample should go some way towards allaying these concerns. Moreover, these reviews suggested that altered mood states may explain some of the effects of tryptophan depletion (Feenstra and van der Plasse 2010; van Donkelaar et al. 2011). As such, we sought to assess the impact of subject mood state on this effect. We have previously shown that mood state can modulate the role of ATD on some, (Robinson et al. 2009) but not all (Robinson and Sahakian 2009b) cognitive tasks. In particular, mood state does not appear to modulate the role of ATD on the inhibition of aversive go/no-go responses (Robinson and Sahakian 2009b). As such, we predicted that mood would not modulate the effect of ATD on affective inhibition, thereby arguing against a universal role of mood in the effects of tryptophan depletion on cognitive processing.

Healthy female subjects $(N=41)$ undergoing either ATD or a balanced (BAL) procedure in a between-subjects design completed the reward and punishment reversal learning task adopted in our first study. Half of each group also received negative, and half neutral, mood induction procedures (MIP). We predicted that ATD would enhance aversive processing by abolishing a positive bias (i.e. increased punishment errors) present under balanced conditions (Cools et al. 2008b), and that this would be insensitive to mood state (Robinson and Sahakian 2009b).

\section{Methods}

Procedures were approved by the Cambridge Research Ethics Committee (06/Q0108/160) and were in accord with the Helsinki Declaration of 1975. All subjects were screened for psychiatric and neurological disorders, gave written informed consent and were compensated for participation. Exclusion criteria were cardiac, hepatic, renal, pulmonary, neurological, psychiatric or gastrointestinal disorders, drug use and personal or family history of any depressive disorder. Subjects were instructed to abstain from alcohol, caffeine and food from midnight prior to each session. Forty-two female subjects (to avoid gender confounds often seen in ATD) were recruited. One subject did not complete testing. The remaining 41 subjects were then divided into four groups: (1) ATD subjects who underwent negative MIP $(N=11)$, (2) ATD subjects who underwent neutral MIP $(N=10)$, (3) BAL subjects who underwent negative MIP $(N=10)$, (4) BAL subjects who underwent neutral MIP $(N=10)$. Subjects completed a number of state and trait measures; the Beck Depression Inventory (BDI; (Beck et al. 1961)), the Impulsiveness, Venturesomeness and Empathy questionnaire (IVE; (Eysenck and Eysenck 1978)) and the Behavioural Inhibition/Behavioural Activation Scale (BIS/BAS (Carver and White 1994)). The groups were demographically matched (see Table 1) and within the same phase of menses $\left(t_{37}=-0.3\right.$, $p=0.8$ ). All subjects also completed a second test session (not presented here, see (Robinson and Sahakian 2009a)).

Table 1 Demographic characteristics

\begin{tabular}{lrrr}
\hline & Mean & S.D. & $P$ \\
\hline Age & 27.6 & 6.4 & 0.3 \\
BDI & 4.5 & 4.1 & 0.4 \\
Impulsiveness & 6.5 & 4.1 & 0.2 \\
Venturesomeness & 8.5 & 3.7 & 0.2 \\
Empathy & 13.5 & 3.7 & 0.8 \\
BIS & 21.1 & 3.4 & 0.4 \\
BAS-drive & 11.7 & 2.1 & 0.2 \\
BAS-fun & 12.3 & 2.1 & 0.8 \\
BAS-reward & 16.9 & 2.0 & 0.9 \\
\hline
\end{tabular}

Non-significant $P$ values indicate a lack of group effect on any of these variables 


\section{Procedure}

Subjects arrived at the research centre between 0830 and 1030 hours and consumed the amino acid mixture. A blood sample was taken, and a nutritionally balanced (BAL) or a tryptophan free (ATD) amino acid drink was ingested. Following this, they were allowed to consume water and were given a low-protein snack (an apple) for lunch. After a resting period of approximately $5 \mathrm{~h}$ to ensure stable and low tryptophan levels (Carpenter et al. 1998), a second blood sample was taken, and the MIP was completed. The MIP was followed by neuropsychological task completion.

Amino acid mixtures

Amino acid mixtures (prepared by SHS international; Liverpool, UK) were identical to previous research (Cools et al. 2008b; Crockett et al. 2009; Robinson and Sahakian 2009a; Roiser et al. 2006): L-alanine, 2.9 g; L-arginine, $2.6 \mathrm{~g}$; L-cystine, $1.4 \mathrm{~g}$; glycine, $1.7 \mathrm{~g}$; L-histidine, $1.7 \mathrm{~g}$; Lisoleucine, $4.2 \mathrm{~g}$; L-leucine, $7.1 \mathrm{~g}$; L-lysine, $4.7 \mathrm{~g}$; Lmethionine, $1.6 \mathrm{~g}$; L-proline, $6.4 \mathrm{~g}$; L-phenylalanine, $3.0 \mathrm{~g}$; L-serine, $3.6 \mathrm{~g}$; L-threonine, $3.4 \mathrm{~g}$; L-tyrosine, $3.6 \mathrm{~g}$; Lvaline, $4.7 \mathrm{~g}$; L-tryptophan, $2.1 \mathrm{~g}$-total BAL, 54.7/TRP, $52.6 \mathrm{~g}$. The drinks were prepared by stirring the mixture into approximately $200 \mathrm{ml}$ tap water with either lemon-lime or grapefruit flavouring. Subjects reported no side effects apart from transient nausea following ingestion of the drink. Blood samples were analysed for the crucial TRP/sum of the long neutral amino acids ( $\Sigma$ LNAA) ratio.

\section{Mood induction procedure}

The MIP (Robinson and Sahakian 2009a, b; Robinson et al. 2009) required subjects to relate negative or neutral Velten sentences to situations in their own lives whilst listening to mood-congruent music. It was programmed in Microsoft Visual Basic 6 and presented on a PaceBlade tablet computer (11-in. monitor). A set of visual analogue scales was administered (T1) at admission, (T2) $5 \mathrm{~h}$ later pre-MIP and (T3) post-MIP to determine self-reported mood.

\section{Neuropsychological testing}

The ability to learn and update reward and punishment predictions was measured using a deterministic reversal learning paradigm employed previously (Cools et al. 2006, 2008b; Robinson et al. 2010). Subjects were presented with two stimuli-one scene and one face. At any given point in time, one stimulus was associated with reward, whilst the other was associated with punishment. On each trial, one stimulus was highlighted by a thick black border and subjects had to predict whether the highlighted stimulus would lead to reward or punishment. They made their prediction by pressing one of two keys (one for punishment, one for reward; order counterbalanced) on a keyboard prior to receiving feedback. Subjects were then shown the actual outcome. Reward consisted of a green smiley face, a ' $+\$ 100$ ' sign and a high-frequency jingle tone. Punishment consisted of a red sad face, a ' $-\$ 100$ ' sign and a single low-frequency tone. The outcomes did not serve as direct performance feedback or as reinforcement. Instead subjects inferred whether their response was correct or not by remembering the key that the pressed (reward or punishment) and comparing it to the actual outcome. The stimulus-outcome contingencies reversed multiple times following attainment of a variable learning criterion (range from five to nine trials). Note that during training, subjects practiced the task until they learned that the outcomes were not performance feedback. However, if they erroneously began to consider the outcomes as performance feedback, they would not reach the learning criterion, and the task would never reverse.

There were two types of experimental condition. In the reward condition, reversals were signalled by providing subjects with unexpected reward following a stimulus previously associated with punishment (i.e. subjects predicted punishment based upon the previous trials, but the stimulus was unexpectedly followed by reward). Conversely, in the punishment condition, reversals were signalled by unexpected punishment. The maximum number of reversal stages per experimental block was 16, although the block terminated automatically after completion of 120 trials (6.6 min). Subjects completed two blocks of reward reversals and two blocks of punishment reversals. Thus, each subject performed 480 trials (four blocks) per experimental session. The order of valance conditions was counterbalanced between subjects.

As previously, performance was assessed by examining proportional errors on four trial types (Cools et al. 2008b): (1) non-reversal reward trials (defined as all trials that required reward prediction, but no updating of stimulusoutcome associations; only trials that followed correct expected outcome predictions were included); (2) nonreversal punishment trials (defined as above, but requiring punishment prediction); (3) reward reversal trials (defined as the trial that followed unexpected reward); (4) punishment reversal trials (defined the trial that followed unexpected punishment). Unexpected reward trials were collapsed across the two unexpected reward blocks (and vice versa for punishment). Reversal and non-reversal trials were assessed using separate repeated measures ANOVAs. Error rates were transformed into proportional scores and arcsine transformed $(2 *$ arcsine $(\sqrt{ } x))$. These transformed proportional scores were submitted to a repeated measures ANOVA with valence condition (two levels: unexpected 
reward and unexpected punishment) and trial-type (three levels: reversal, non-reversal reward and non-reversal punishment) as within-subjects factors and drink (two levels) and mood (two levels) as between-subjects factors. Simple effects analyses were Bonferroni corrected.

\section{Results}

Amino acid mixtures

Amino acid data (Table 2) were incomplete for three (one ATD and two BAL) participants due to blood extraction problems. A repeated measures ANOVA revealed a significant two-way drink $\times$ time interaction for the critical TRP/ $\Sigma$ LNAA ratio (treatment $\times$ time; $F_{1,36}=66 ; P<0.001$ ). Simple effects analyses revealed that the significant drink $\times$ time interaction was due to a $92 \%$ decrease in the TRP/ $\Sigma$ LNAA ratio following ATD (significant effect of time, $\left.F_{1,36}=42 ; P<0.001\right)$ but a $92 \%$ increase in the TRP $/ \Sigma L N A A$ ratio following $\operatorname{BAL}\left(F_{1,36}=26 ; P<0.001\right)$.

\section{Mood change}

There was no change in sad mood state between the start of the experiment (T1) and $5 \mathrm{~h}$ later (T2) in either the ATD $\left(F_{1,35}=2\right)$ or BAL $\left(F_{1,35}=1\right)$ group (Table 2$)$. However, there was a significant increase in sad mood after the sad MIP (T3) relative to (T2) $\left(F_{1,36}=11, P=0.003\right)$, but not before and after the neutral MIP $\left(F_{1,36}=1\right)$ (Table 2). Thus, the MIP, but not the drink, altered participant's mood state.

\section{Neuropsychological testing}

Pooling all trial types (reversal, non-reversal reward and non-reversal punishment) together revealed a significant drink (ATD, BAL) by valence condition (reward, punishment) interaction $\left(F_{1,35}=4.7, P=0.04\right)$. There was also a significant valence condition by trial type (reversal, nonreversal reward block, non-reversal punishment block) interaction $\left(F_{2,34}=3.5, P=0.04\right)$. As such, in line with our

Table 2 Mood ratings

\begin{tabular}{lllll}
\hline Manipulation & Before & \multicolumn{3}{c}{ After } \\
\hline ATD & 5.8 & $(0.4)$ & 5.2 & $(0.4)$ \\
BAL & 5.6 & $(0.4)$ & 5.5 & $(0.4)$ \\
Negative MIP & 5.2 & $(0.4)$ & 4.0 & $(0.4)$ \\
Neutral MIP & 5.5 & $(0.4)$ & 5.3 & $(0.4)$ \\
\hline
\end{tabular}

Distance (centimetre) marked along a 10 -cm visual analogue scale asking 'how happy are you?' Treatment effects are assessed prior to the MIP, and MIP effects are collapsed across treatment sessions previous study, we next examined the reversal and nonreversal stages separately.

Non-reversal trials Non-reversal errors (Table 3) were examined using an ANOVA with trial type (non-reversal reward, non-reversal punishment) and valence condition (reward reversal, punishment reversal) as within-subjects factors, and drink (ATD, BAL) and MIP (neutral, negative) as between-subjects factors. Consistent with our previous study (Cools et al. 2008b), there was a significant interaction between drink and trial type $\left(F_{1,35}=4.4, P=0.04\right)$, which was driven by a significant effect of drink on punishment non-reversal trials $\left(F_{1,35}=4.3, P=0.04\right)$, but not on reward non-reversal trials $\left(F_{1,35}=1.0\right)$. Moreover, subjects made significantly more errors on punishment than reward nonreversal trials after the BAL drink $\left(F_{1,35}=15.4, P<0.001\right)$ while there was no difference between punishment and reward non-reversal trials after ATD $\left(F_{1,35}=1.3\right.$; Fig. 1$)$. This effect was, however, robust across mood state (no MIP $\times$ trial type $\times$ outcome interaction, $\left.F_{1,35}=0.009\right)$ and valence condition (no valence condition $\times$ trial type $\times$ outcome interaction, $\left.F_{1,35}=0.007\right)$.

Reversal trials Reversal errors (Table 3) were examined in an ANOVA with valence condition (reward reversal, punishment reversal) as a within-subject factor and drink (ATD, BAL) and MIP (neutral, negative) as between-subjects factors. Consistent, again, with our prior study, there was no drink by valence condition interaction $\left(F_{1,35}=3.1\right)$. Moreover, this effect was robust across mood state (MIP $\times$ drink $\times$ valence condition interaction, $F_{1,35}=0.1$ ).

Thus, as before, ATD abolished a disproportionate difficulty with punishment prediction, but did not affect reward prediction. This effect was restricted to the nonreversal trials, and was robust across mood states.

\section{Discussion}

The present results demonstrate that acute tryptophan depletion abolishes a disproportionate inability to predict punishment which is present following consumption of a balanced amino acid drink. This replicates our previous finding (Cools et al. 2008b) in an independent and larger sample of subjects and using a different between-subjects experimental design. Moreover, the finding extends prior work by suggesting that this effect on punishment prediction, unlike more complex cognitive effects of tryptophan depletion (Robinson et al. 2009), may be impervious to subject mood state.

Previously, we postulated that the ATD-induced improvement in punishment prediction might represent enhanced 
Table 3 Error rates

\begin{tabular}{|c|c|c|c|c|c|c|c|c|}
\hline \multirow[b]{3}{*}{ Group } & \multicolumn{8}{|c|}{ Non-reversal } \\
\hline & \multicolumn{4}{|c|}{ Reward } & \multicolumn{4}{|c|}{ Punishment } \\
\hline & ATD- & $\mathrm{ATD}=$ & BAL- & $\mathrm{BAL}=$ & ATD- & $\mathrm{ATD}=$ & BAL- & $\mathrm{BAL}=$ \\
\hline Mean & 0.036 & 0.037 & 0.058 & 0.037 & 0.037 & 0.046 & 0.116 & 0.074 \\
\hline \multirow[t]{2}{*}{ SEM } & 0.011 & 0.010 & 0.011 & 0.011 & 0.028 & 0.027 & 0.030 & 0.030 \\
\hline & \multicolumn{8}{|c|}{ Reversal } \\
\hline Mean & 0.096 & 0.089 & 0.070 & 0.119 & 0.033 & 0.043 & 0.076 & 0.135 \\
\hline SEM & 0.038 & 0.037 & 0.041 & 0.041 & 0.045 & 0.043 & 0.048 & 0.048 \\
\hline
\end{tabular}

$A T D$ acute tryptophan depletion, $B A L$ balanced drink, - negative mood induction, = neutral mood induction. $(\mathrm{ATD}-: n=10, \mathrm{ATD}=: n=11, \mathrm{BAL}-$ : $n=9 ; \mathrm{BAL}=: n=9), S E M$ standard error of the mean

aversive temporal-difference prediction error signals, brought about by lowering tonic 5-HT levels and increasing the signal-to-noise ratio of phasic 5-HT bursts (Cools et al. 2008b). In light of recent theoretical (Dayan and Huys 2008, 2009) and experimental approaches (Crockett et al. 2009), however, an alternative explanation might be that reducing serotonin attenuated punishment-induced inhibition. In the theoretical model proposed by Dayan and Huys (2008), serotonin is said to 'prune' aversive branches of 'the decision tree', leading to a neglect of aversive thoughts, and then subsequent actions, under optimal serotonin levels. Specifically, the increased non-reversal errors following the balanced drink may be a result of subjects successfully inhibiting punishment-related thoughts and actions, and hence making increased errors to expected punishment cues under balanced serotonin conditions. Reducing tryptophan (and hence putatively serotonin) may serve to remove this punishment-linked inhibition, releasing punishment responses, and bringing punishment errors down to the same level as reward errors. Errors following unexpected outcomes may, moreover, be impervious to this effect because (a) inhibitory processes may take more than one trial to be implemented (and reversal analysis only examines errors on the one trial following unexpected feedback) and/or (b) reversal trials require response switching which may mask any inhibitory effects.

Regardless of the interpretation, the present finding may be critical for our understanding of resilience to affective disorders. Specifically, increased inhibition of aversive thoughts may be an adaptive means to reduce the neuropsychological impact of aversive events which promotes, to a certain extent, blissful ignorance (Alloy and Abramson 1979; Robinson et al. 2011) and cushions against stress. Such inhibition might be removed by serotonin reduction (Cools et al. 2008a) and, in more naturalistic environments, via the recruitment of stressspecific neural networks (e.g. the amygdala (Cools et al. 2005)). Indeed both ATD and induced stress (via threat of shock) restore aversive processing which is reduced under non-anxious conditions (Robinson et al. 2011). As such, on the flipside of the same coin, optimal levels of serotonin might promote resilience to affective disorders under non-anxious conditions by inhibiting responses to aversive stimuli.

Our failure to see an interaction between mood and ATD suggests that effects of ATD do not universally depend on mood. The previous demonstration of an interaction between mood and ATD (Robinson et al. 2009) was seen in two complex tasks which required integration of information from multiple domains (i.e. self-referent recall and integrating pattern recognition with cued reinforcement) (Robinson et al. 2009), but was not seen during affective inhibition (Robinson and Sahakian 2009b). As such, we previously argued (Robinson and Sahakian 2009b) that the mediating effect of mood may be restricted to tasks which require complex and integrative cortical processing rather than simple 'bottom up' activation of subcortical structures like the amygdala and striatum (which may be more basic, primal and fast responses that are not modulated by mood), but this is of course speculative and requires further study. It is notable that both tasks that fail to show an interaction involve affective inhibition, but of course the possibility that inadequate

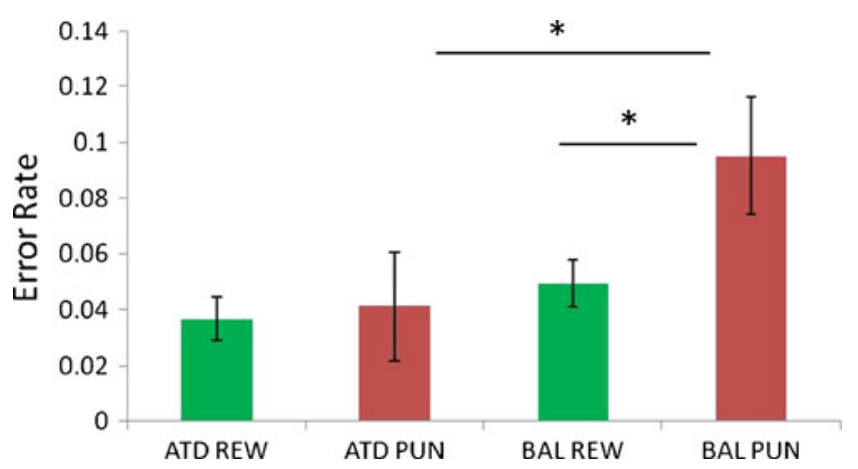

Fig. 1 Tryptophan depletion removes a significant impairment in non-reversal punishment prediction which was present under the balanced condition. Scores represent untransformed error proportions, error bars are standard error of the mean; ${ }^{*} P<0.05$. ATD acute tryptophan depletion, $B A L$ balanced drink, REW reward, $P U N$ punishment 
sample sizes account for the lack of effect should not be ruled out. It should be noted, furthermore, that the present mood induction technique was also adopted in both the study demonstrating an interaction (Robinson et al. 2009), and the study failing to show an interaction (Robinson and Sahakian 2009b). As such, it is difficult to attribute this null effect to a failure of the manipulation. This finding thus argues against a universal role of mood state (Crockett et al. in submission) (e.g. negative mood associated with the procedure) in the effects of tryptophan depletion on cognitive processing (Feenstra and van der Plasse 2010; van Donkelaar et al. 2011).

It should also be noted that whilst the previous effect was seen within subjects, this study demonstrated the same effect between subjects. The present design has the advantage of ruling out order and sequence effects associated with repeating the same task in the same subjects, but it requires a larger sample size to achieve an equivalent level of sensitivity. Demonstrating remarkably similar effects with such different designs serves to enhance our confidence in the validity of our initial findings (Cools et al. 2008b).

The BAL group demonstrated a significant increase in tryptophan levels over the course of the day. This effect is seen in some (Crockett et al. 2009; Robinson and Sahakian 2009b; Robinson et al. 2009; Roiser et al. 2006) but not all previous studies (Cools et al. 2008b; Robinson et al. 2009). One implication of this observation is that we cannot precisely specify the direction of the effect. It is possible that tryptophan depletion enhanced punishment processing, as has been argued previously (Cools et al. 2008a; Crockett et al. 2009), but it is also possible that tryptophan supplementation led to impaired punishment (relative to reward) processing, as has also been shown (Murphy et al. 2006, 2008). If the latter, it could argue for the use of tryptophan supplementation as a means to promote resilience in healthy individuals (Elliott et al. 2010; Murphy et al. 2008), or to increase inhibition in disorders of impulsivity and compulsivity, such as attention deficit hyperactivity disorder (ADHD) and obsessive-compulsive disorder (OCD). Nevertheless, in our previous study with the same task, the same effect was seen under BAL despite no change of tryptophan ratios in this group (Cools et al. 2008 b), so caution should be taken with this interpretation. Moreover, it is important to recognise that this ambiguity does not invalidate the central hypothesis that serotonin is involved in aversive inhibition. The hypothesis predicts increased inhibition following serotonin increases, as well as decreased inhibition following serotonin decreases. Nevertheless, this inability to specify the direction of the effect is a major weakness of the study. Future research might consider adding an additional baseline session without pharmacological manipulation to address this issue more directly.

\section{Conclusion}

These findings demonstrate the facilitatory effects of ATD on punishment (and not reward) prediction, but extend them by showing them to be impervious to mood state. We argue that the effect may be due to tryptophan depletion removing serotonin-mediated inhibition of aversive thoughts, and hence actions, and that this may, moreover, reduce the psychological impact of negative life events and promote resilience to affective disorders. Disorders in which aversive processing is enhanced, such as anxiety and depression, as well as those in which inhibition is reduced, such as OCD and ADHD, are economically and emotionally costly to the individual and society. Increasing our understanding of how it might be possible to cushion against such disorders, potentially by enhancing behavioural inhibition to aversive stimuli, would go a long way towards improving the 'mental wealth of nations' (Beddington et al. 2008).

Open Access This article is distributed under the terms of the Creative Commons Attribution Noncommercial License which permits any noncommercial use, distribution, and reproduction in any medium, provided the original author(s) and source are credited.

\section{References}

Alloy LB, Abramson LY (1979) Judgment of contingency in depressed and nondepressed students: sadder but wiser? J Exp Psychol Gen 108:441-485

Beck AT, Ward CH, Mendelson M, Mock J, Erbaugh J (1961) An inventory for measuring depression. Arch Gen Psychiatr 4:561571

Beddington J, Cooper CL, Field J, Goswami U, Huppert FA, Jenkins R, Jones HS, Kirkwood TBL, Sahakian BJ, Thomas SM (2008) The mental wealth of nations. Nature 455:1057-1060

Boureau YL, Dayan P (2011) Opponency revisited: competition and cooperation between dopamine and serotonin. Neuropsychopharmacology 36:74-97

Carpenter LL, Anderson GM, Pelton GH, Gudin JA, Kirwin PD, Price LH, Heninger GR, McDougle CJ (1998) Tryptophan depletion during continuous CSF sampling in healthy human subjects. Neuropsychopharmacology 19:26-35

Carver CS, White TL (1994) Behavioral inhibition, behavioral activation, and affective responses to impending reward and punishment: the BIS/BAS Scales. J Personal Soc Psychol 67:319-333

Cools R, Calder A, Lawrence A, Clark L, Bullmore E, Robbins T (2005) Individual differences in threat sensitivity predict serotonergic modulation of amygdala response to fearful faces. Psychopharmacology 180:670-679

Cools R, Lewis SJG, Clark L, Barker RA, Robbins TW (2006) LDOPA disrupts activity in the nucleus accumbens during reversal learning in Parkinson's disease. Neuropsychopharmacology $32: 180-189$

Cools R, Roberts AC, Robbins TW (2008a) Serotoninergic regulation of emotional and behavioural control processes. Trends Cogn Sci $12: 31-40$ 
Cools R, Robinson OJ, Sahakian BJ (2008b) Acute tryptophan depletion in healthy volunteers enhances punishment prediction but does not affect reward prediction. Neuropsychopharmacology 33:2291-2299

Cools R, Nakamura K, Daw ND (2011) Serotonin and dopamine: unifying affective, activational, and decision functions. Neuropsychopharmacology 36:98-113

Crockett MJ, Clark L, Robbins TW (2009) Reconciling the role of serotonin in behavioral inhibition and aversion: acute tryptophan depletion abolishes punishment-induced inhibition in humans. J Neurosci 29:11993-11999

Daw ND, Kakade S, Dayan P (2002) Opponent interactions between serotonin and dopamine. Neural Netw 15:603-616

Dayan P, Huys QJM (2008) Serotonin, inhibition, and negative mood. PLoS Comput Biol 4:e4

Dayan P, Huys QJM (2009) Serotonin in affective control. Annu Rev Neurosci 32:95-126

Deakin JFW, Graeff FG (1991) 5-HT and mechanisms of defence. J Psychopharmacol 5:305-315

Elliott R, Sahakian BJ, Charney D (2010) The neural basis of resilience. In: Cooper CL, Goswami U, Sahakian BJ (eds) Mental Capital and Wellbeing. Wiley-Blackwell

Eysenck SB, Eysenck HJ (1978) Impulsiveness and venturesomeness: their position in a dimensional system of personality description. Psychol Rep 43:1247-1255

Feenstra MGP, van der Plasse G (2010) Tryptophan depletion and serotonin release - a critical reappraisal. In: Christian PM, Barry LJ (eds) Handbook of behavioral neuroscience. Elsevier, pp 249-258

Gessa GL, Biggio G, Fadda F, Corsini GU, Tagliamonte A (1974) Effect of the oral administration of tryptophan-free amino acid mixtures on serum tryptophan, brain tryptophan and serotonin metabolism. J Neurochem 22:869-870

Murphy S, Longhitano C, Ayres R, Cowen P, Harmer C (2006) Tryptophan supplementation induces a positive bias in the processing of emotional material in healthy female volunteers. Psychopharmacology 187:121-130

Murphy SE, Longhitano C, Ayres RE, Cowen PJ, Harmer CJ, Rogers $\mathrm{RD}$ (2008) The role of serotonin in nonnormative risky choice: the effects of tryptophan supplements on the "reflection effect" in healthy adult volunteers. J Cogn Neurosci 21:1709-1719

Robinson OJ, Sahakian BJ (2009a) Acute tryptophan depletion evokes negative mood in healthy females who have previously experienced concurrent negative mood and tryptophan depletion. Psychopharmacology 205:227-235

Robinson OJ, Sahakian BJ (2009b) A double dissociation in the roles of serotonin and mood in healthy subjects. Biol Psychiatry 65:89-92

Robinson OJ, Cools R, Crockett MJ, Sahakian BJ (2010a) Mood state moderates the role of serotonin in cognitive biases. J Psychopharmacol 24(4):573-583, 0269881108100257

Robinson OJ, Standing H, DeVito E, Cools R, Sahakian BJ (2010b) Dopamine precursor depletion improves punishment prediction during reversal learning in healthy females but not males. Psychopharmacology 211:187-195

Robinson OJ, Letkiewicz A, Overstreet C, Ernst M, Grillon C (2011) The effect of induced anxiety on cognition: threat of shock enhances aversive processing in healthy individuals. Cogn Affect Behav Neurosci 11(2):217-227

Roiser JP, Müller U, Clark L, Sahakian BJ (2006) The effects of acute tryptophan depletion and serotonin transporter polymorphism on emotional processing in memory and attention. Int $\mathrm{J}$ Neuropsychopharmacol 10:449-461

Soubrié P (1986) Reconciling the role of central serotonin neurons in human and animal behaviour. Behav Brain Sci 9:319-364

van Donkelaar EL, Blokland A, Ferrington L, Kelly PAT, Steinbusch HWM, Prickaerts J (2011) Mechanism of acute tryptophan depletion: is it only serotonin? Mol Psychiatr 16 (7):695-713

Williams WA, Shoaf SE, Hommer D, Rawlings R, Linnoila M (1999) Effects of acute tryptophan depletion on plasma and cerebrospinal fluid tryptophan and 5-hydroxyindoleacetic acid in normal volunteers. J Neurochem 72:1641-1647

Young SN, Gauthier S (1981) Effect of tryptophan administration on tryptophan, 5-hydroxyindoleacetic acid and indoleacetic acid in human lumbar and cisternal cerebrospinal fluid. J Neurol Neurosurg Psychiatr 44:323 